\title{
Ultracold atoms interacting with a sinusoidal mode of a high $Q$ cavity
}

\author{
J. C. Retamal ${ }^{\dagger}$, E. Solano ${ }^{\dagger *}$ and N. Zagury* \\ $\dagger$ Departamento de Física, Universidad de Santiago de Chile, Casilla 30\%, Correo 2, Santiago, Chile \\ ${ }^{\dagger}$ Seccion Física, Departamento de Ciencias, Pontificia Universidad Catolica del Peru, Ap. 1761, Lima, Peru \\ * Instituto de Física, Universidade Federal do Rio de Janeiro, Caixa Postal 68528, 21945-970 Rio de Janeiro, RJ, Brazil
}

(August 21, 2018)

\begin{abstract}
We consider the interaction of two level ultracold atoms resonant with a sinusoidal mode of the electromagnetic field in a high $Q$ cavity. We found that well resolved resonances appear in the transmission coefficients even for actual interaction and cavity parameters. The probability of emission of one photon and the probability of transmission of an atom, when number or coherent states are initially present in the cavity, are discussed. The interplay between the increasing width of the resonances and multi-peak steady-state photon-statistics are also studied. Furthermore, we compare our results with those of a constant field mode.
\end{abstract}

PACS numbers: 42.50.-p, 32.80.-t, 42.50.Ct, 42.50.Dv

\section{INTRODUCTION}

Recent developments in cooling of neutral atoms [1] have called the attention on the interaction of ultracold atoms with microwave cavities [2 7]. Very cold atoms (kinetic energies much smaller than the atom-field interaction energy ) have a peculiar behavior when interacting with a cavity, they are strongly reflected unless the ratio of the size of the cavity and the atomic wave length associated to the interaction energy are close to certain resonant values. For example, it has been shown [3,5] that ultracold atoms of mass $m$ may be totally reflected by a cavity of size $L$ containing $n$ photons, in a constant field mode, unless the Rabi frequency $\Omega_{n}$ is close to $2 \hbar(j \pi / L)^{2} /(2 m), j=0,1,2 \ldots$, when only approximately half of the atoms are reflected.

Models for the interaction of atoms with a microwave cavity usually do not take into account the spatial dependence of the interaction coming from the electric field profile [8]. This is justified because, in general, we are interested in the atom-field state after the atom has crossed the cavity and are dealing with atomic thermal velocities, which are exceedingly high compared with the recoil ones experienced by the atom inside the cavity.

In order that we may observe any effect on the centerof-mass motion of the atoms, their velocities have to be reduced to very low values in such a way that even the microscopic inversion of momentum of the center-of-mass could take place. Actually, the only way for an atom to experience mechanical action on its center-of-mass is through the mechanism of exchange of momentum with the whole cavity. At high atomic velocities the energy exchange between the field and the atoms takes place through the Rabi flopping of the internal atomic levels. At very low atomic velocities the spatial variation of the electromagnetic field acting on the atoms provides an additional driving mechanism which lies in the momentum exchange of the atom with the cavity.

The aim of the present work is to investigate the effect of a sinusoidal electric field mode when ultracold atoms are sent into a cylindrical microwave cavity for actual experimental parameters. We will show that under these conditions the behavior of the relevant physical quantities may be quite different from those of a discontinuous field mode, although still preserving resonant features if the atoms are cold enough.

\section{THE MODEL}

Let us assume that a two level atom is travelling along the $z$-direction in the way of a microwave high $Q$ cavity of length $L$, containing a quantized field mode in resonance with the two level atomic transition $|e\rangle \Leftrightarrow|f\rangle$.

The Hamiltonian describing the motion of the two level atom in the $z$-direction is given, in the interaction representation, by

$$
H=\frac{p^{2}}{2 m}+\frac{\hbar^{2} \kappa^{2}}{2 m} u(z)\left(a \sigma^{\dagger}+a^{\dagger} \sigma\right) .
$$

Here $\sigma \equiv|f\rangle\langle e|, a\left(a^{\dagger}\right)$ is the annihilation (creation) operator of the electromagnetic field mode whose spatial modulation pattern is $u(z)$ and $\hbar \kappa^{2} /(2 m)$ is half the Rabi frequency associated with the transition $|f\rangle \leftrightarrow|e\rangle$ in the vacuum. $u(z)$ is normalized in such a way that the area under the mode is $L$. In refs. 3,5 $u(z)$ is taken as a square function of height 1 and width $L$ (mesa function). In this case it can be shown that the transmission of ultracold atoms through a cavity containing $n$ photons is very small unless

$$
\kappa_{n} \equiv \kappa \sqrt[4]{n+1}
$$

is a multiple of $\pi / L$. In ref. [6] the $\operatorname{sech}^{2}(z / L)$ and sinusoidal modes were considered and the authors have concluded that, for high values of $L$, the resonances referred 
above are smeared out. Here we will discuss in more detail the sinusoidal mode having just one antinode. We will show that in this case, resonances still exist for high values of $\kappa_{n} L$ as long as the atoms are cold enough.

The Hamiltonian given in Eq. 11 can be conveniently written in the dressed state basis as:

$$
H=\sum\left[\frac{p^{2}}{2 m} \pm V_{n}(z)\right]| \pm, n\rangle\langle \pm, n|,
$$

where $V_{ \pm n}(z)$ is given by

$$
V_{ \pm n}(z)= \pm \frac{\hbar^{2} \kappa_{n}^{2}}{2 m} u(z)
$$

and

$$
| \pm, n\rangle=(|e, n\rangle \pm|f, n+1\rangle) / \sqrt{2} \text {. }
$$

In the dressed state basis our problem is equivalent to that of a particle being scattered by the potentials $V_{ \pm n}(z)$. The cavity acts as a barrier when the atom-field state is $|+n\rangle$ and acts as a well when the atom-field state is $|-n\rangle$.

Before the atom reaches the cavity containing the field $\sum c(n)|n\rangle$, the initial atomic state is given by the product of a wave packet in momentum space $\langle z \mid \phi\rangle=\int d k \phi(k) e^{i k z}$ times the internal energy state of the atom that, for definitiveness, we assume to be the upper state $|e\rangle$. After the atom interacts with the cavity the total atom-cavity state is (for $|z|>L / 2$ ):

$\sum c(n) \int d k \phi(k) \exp \left(-i \frac{\hbar^{2} k^{2}}{2 m} \tau\right)\left[\left(e^{i k z} t_{+n}(k)+e^{-i k z} r_{+n}(k)\right)\right.$
$\left.|+n\rangle+\left(e^{i k z} t_{-n}(k)+e^{-i k z} r_{-n}(k)\right)|-n\rangle\right]$

where the reflection, $r_{ \pm n}$, and transmission, $t_{ \pm n}$, coefficients are associated with the scattering eigenfunctions $\varphi_{k}^{ \pm}(z)$. These functions are solutions of the time independent Schrödinger equation :

$$
\left[\frac{d^{2}}{d z^{2}}+k^{2} \mp \kappa_{n}^{2} u(z)\right] \varphi_{k}^{ \pm}(z)=0
$$

where $\hbar k$ is the momentum of the incident particle.

In this paper we are interested in discussing a situation where the longitudinal $z$-dependence of the electric field is sinusoidal:

$$
u(z)=\theta(L / 2-|z|)(\pi / 2) \cos \left(\frac{\pi z}{L}\right) .
$$

This is the case of a cylindrical cavity tuned either to a $\mathrm{TE}_{m n 1}$ or a $\mathrm{TM}{ }_{m n 1}$ mode [9].

We may try to obtain the values of $r_{ \pm n}(k)$ and $t_{ \pm n}(k)$ by numerically integrating a complex solution of Eq. for runaway asymptotic conditions. An alternative way, where we found numerical and analytical advantages, is to consider instead the even $\left(\varphi_{e}^{ \pm n}(z)\right)$ and odd $\left(\varphi_{o}^{ \pm n}(z)\right)$ real (up to an overall complex constant) eigensolutions of Eq. 7, such that for $z<-L / 2$ [10]

$$
\varphi_{e, o}^{ \pm n}(z) \propto \cos \left(k z-\delta_{e, o}^{ \pm n}\right) .
$$

Here $\delta_{e}^{ \pm n}(k)\left(\delta_{o}^{ \pm n}(k)\right)$ is the phase shift associated to the even (odd) eigensolution of the Schrödinger equation. In terms of these phase shifts the reflection and transmission coefficients may be written as

$$
r_{ \pm n}(k)=\frac{e^{2 i \delta_{e}^{ \pm n}}+e^{2 i \delta_{o}^{ \pm n}}}{2}
$$

$$
t_{ \pm n}(k)=\frac{e^{2 i \delta_{e}^{ \pm n}}-e^{2 i \delta_{o}^{ \pm n}}}{2} .
$$

The phase shifts are related to the logarithmic derivatives at $z=-L / 2$ by

$$
\beta_{e, o}^{ \pm n}=k \tan \left(\kappa_{n} L / 2+\delta_{e, o}^{ \pm n}\right)
$$

Notice that $\beta_{e, o}^{ \pm n}$ are real in contrast with the complex logarithmic derivative of a typical, right or left, propagating solution. ¿From Eqs. 10 and 11 it is easy to show that:

$$
r_{ \pm n}(k)=\frac{k^{2}+\beta_{e}^{ \pm n} \beta_{o}^{ \pm n}}{\left(k-i \beta_{e}^{ \pm n}\right)\left(k-i \beta_{o}^{ \pm n}\right)} e^{-i k L}
$$

and

$$
t_{ \pm n}(k)=\frac{i k\left(\beta_{e}^{ \pm n}-\beta_{o}^{ \pm n}\right)}{\left(k-i \beta_{e}^{ \pm n}\right)\left(k-i \beta_{o}^{ \pm n}\right)} e^{-i k L} .
$$

In the case of the potential barrier, for $\kappa_{n} L \gg 1$ and $k \ll \kappa_{n}$, the transmission coefficient $t_{+n}$ is very small 6) and close to zero. In the case of the potential well, and for $k \ll \kappa_{n}$ the transmission coefficient $t_{-n}$ is close to zero unless $\beta_{e}^{-n} \beta_{o}^{-n}=-k^{2}$, that is for very small $\beta_{e}^{-n}$ or very small $\beta_{o}^{-n}$. For a fixed value of $k / \kappa_{n}$, these resonances in the transmission coefficient occur at special values of $\kappa_{n} L$, which would correspond to the appearance of a bound state with $k^{2}=0$ ( notice that the transmission coefficient has a pole at $\left.k=i \beta_{e, o}^{-n}\right)$.

Assume that initially there are $n$ photons inside the cavity and the atom is incident from the left in the upper state $|e\rangle$. After the atom interacts with the cavity, it can be transmitted in the upper state, transmitted in the lower state, reflected in the upper state or reflected in the lower state. The probabilities that these events occur are denoted by $T_{e}^{n}, T_{f}^{n}, R_{e}^{n}$ and $R_{f}^{n}$, respectively and given by:

$$
T_{e}^{n}=\frac{1}{4}\left|t_{+n}+t_{-n}\right|^{2}, \quad T_{f}^{n}=\frac{1}{4}\left|t_{+n}-t_{-n}\right|^{2}
$$

and

$$
R_{e}^{n}=\frac{1}{4}\left|r_{+n}+r_{-n}\right|^{2}, \quad R_{f}^{n}=\frac{1}{4}\left|r_{+n}-r_{-n}\right|^{2} .
$$

Of course $T_{e}^{n}+T_{f}^{n}+R_{e}^{n}+R_{f}^{n}=1$. We will show in the next section that for $k / \kappa_{n} \ll 1, t_{+n} \cong 0$. In this 
case $T_{e}^{n} \approx T_{f}^{n} \approx\left|t_{-n}\right|^{2} / 4$. Due to this last approximate relation and the unitarity condition we choose to discuss below only two quantities of physical interest: the probability of emission of a photon, $P_{\mathrm{em}}^{n}=T_{f}^{n}+R_{f}^{n}$, and the probability of transmission of an atom, $T^{n}=T_{e}^{n}+T_{f}^{n}$. We notice also that when $r_{-n} \cong 0, P_{\mathrm{em}}^{n} \cong T^{n}$.

If we initially have a photon number distribution given by $\left|c_{n}\right|^{2}$, the total probability of photon emission is $P_{\mathrm{em}}=\sum\left|c_{n}\right|^{2} P_{\mathrm{em}}^{n}$ and the total probability of atomic transmission is $T=\sum\left|c_{n}\right|^{2} T^{n}$. These quantities should be easily measurable by detecting the internal energy levels of the reflected and transmitted atoms. As we will show, these quantities depend strongly on the shape of the field inside the cavity, as long as $k / \kappa_{n} \ll 1$.

\section{ANALYTICAL RESULTS}

The constant field mode $(u(z)=1$, for $|z|<L / 2)$ have been discussed in refs. [2, 3,5]. For $k / \kappa_{n} \ll 1$ we have $\beta_{e}^{+n} \approx-\kappa_{n} \tanh \left(\kappa_{n} L / 2\right), \beta_{o}^{+n} \approx-\kappa_{n} \operatorname{coth}\left(\kappa_{n} L / 2\right)$, $\beta_{e}^{-n} \approx \kappa_{n} \tan \left(\kappa_{n} L / 2\right)$ and $\beta_{o}^{-n} \approx \kappa_{n} \cot \left(\kappa_{n} L / 2\right)$, so that the resonances appear when $\kappa_{n} L$ equals to an integer multiple of $\pi$ [2,3,5]. In this case the resonances are very sharp, their width being approximately constant and equal to $4 k / \kappa_{n}$ even for large $\kappa_{n} L$.

In the case of the sinusoidal mode, as long as $\kappa_{n} L$ is small, it is easy to obtain the even and odd eigensolutions of Eq. 7 by numerical integration. This can be done easily and with confidence for $\kappa_{n} L \lesssim 100 \pi$. If we wish to predict values closer to real experimental situations we need to consider higher values of $\kappa_{n} L$, which are typically of the order of $O\left(10^{5}-10^{6}\right)$ for Rydberg atoms. In these cases the numerical solutions do not converge rapidly for the $|-n\rangle$ channel and we found easier, and more instructive, to use a WKB-like approximation for calculating the $\delta_{e}^{ \pm n}$ and $\delta_{o}^{ \pm n}$ phase shifts or, equivalently, the logarithmic derivatives $\beta_{e, o}^{ \pm n}$.

In the case of the potential barrier, and for $k / \kappa_{n} \ll 1$, we may use the WKB approximation [11] for calculating $\beta_{o, e}^{+n}$. We get

$$
\beta_{e, o}^{+n} \cong-k(1 \mp \Theta),
$$

where the - and + signs inside the parenthesis refer to the even and odd solution and

$$
\Theta=\exp \left(-\int_{-a}^{a} \sqrt{-k^{2}+\kappa_{n}^{2} u(z)} d z\right)
$$

where $\pm a$ are the turning points. Substituting the values for $\beta_{o, e}^{+n}$ in Eqs. 12 and 13 we get $r_{+n}(k) \simeq-i e^{-i k L}$ and $t_{+n}(k) \simeq i \Theta e^{-i k L}$. For $k / \kappa_{n} \ll 1, a \simeq L / 2$ and $\Theta \simeq \exp \left(-4.19 \kappa_{n} L\right)$, which is very small for $\kappa_{n} L>1$.

Inside the cavity the solutions $\varphi_{e, o}^{-n}(z)$ for the potential well may be written, whenever the semiclassical approximation holds, as

$$
\begin{aligned}
& \varphi_{e}^{-n}(z) \approx \frac{1}{\sqrt{q(z)}} \cos \left(\int_{0}^{z} q\left(z^{\prime}\right) d z^{\prime}\right) \\
& \varphi_{o}^{-n}(z) \approx \frac{1}{\sqrt{q(z)}} \sin \left(\int_{0}^{z} q\left(z^{\prime}\right) d z^{\prime}\right)
\end{aligned}
$$

where $q(z)=\sqrt{k^{2}+\kappa_{n}^{2} u(z)}$. It is well known that Eqs. 18 are valid when the condition

$$
\left|\frac{d q(z)}{d z}\right| \ll q(z)^{2}
$$

is fulfilled [11. This condition may be satisfied for the sinusoidal mode and for any $z$, whenever

$$
\xi \equiv \frac{\pi^{2}\left(\kappa_{n} / k\right)^{3}}{4 \kappa_{n} L} \ll 1 .
$$

In this case the semiclassical solutions (Eqs. 18) are valid for all $z$ and the reflection coefficient $r_{-n}(k)$ vanishes as $\xi$ goes to zero, as we will show below. When $\xi \gtrsim 1$ the inequality 19 is not valid near the regions $|z| \lesssim L / 2$. In this case the modulus of $r_{-n}(k)$ may increase even to 1. Notice that we may have $\xi>1$ even for large $k L$ and $\kappa_{n} L$. For example, for $\kappa_{n} L \sim 10^{5}$ and $k L \sim 10^{3}, \xi \approx 24$.

Near $z=-L / 2, u(z)$ may be approximated by a straight line and the solutions of Eq. 7 may be written as:

$$
\varphi_{e, o}^{-n}(z) \propto w^{1 / 3}\left(A_{e, o} J_{1 / 3}(w)+B_{e, o} J_{-1 / 3}(w)\right)
$$

where

$$
w=\frac{\pi}{6} \kappa_{n} L\left(\frac{2 z}{L}+1+\frac{4 k^{2}}{\pi^{2} \kappa_{n}^{2}}\right)^{3 / 2} .
$$

The logarithmic derivatives at $z=-L / 2$ are given by:

$$
\beta_{e, o}^{-n}=k\left(\xi+\frac{A_{e, o} J_{1 / 3}^{\prime}\left(\xi^{-1} / 3\right)+B_{e, o} J_{-1 / 3}^{\prime}\left(\xi^{-1 / 3}\right)}{A_{e, o} J_{1 / 3}\left(\xi^{-1} / 3\right)+B_{e, o} J_{-1 / 3}\left(\xi^{-1} / 3\right)}\right) .
$$

By connecting the even and odd solutions of Eq. 21 with those given by Eqs. 18, we obtain :

$$
\frac{A_{e}}{B_{e}}=\chi(\varphi)=-\frac{\sin (\varphi+\pi / 12)}{\cos (\varphi-\pi / 12)}
$$

and $A_{o} / B_{o}=\chi(\varphi+\pi / 2)$, where

$$
\varphi \simeq \int_{0}^{-L / 2} \sqrt{k^{2}+\kappa_{n}^{2} u(z)} d z
$$

For $\xi \ll 1$, we use the asymptotic expressions for $J_{ \pm 1 / 3}\left(\xi^{-1} / 3\right)$ to obtain $\beta_{e}^{-n} \approx-k(\xi / 2+\tan \varphi)$ and $\beta_{o}^{-n} \approx-k(\xi / 2-\cot \varphi)$. From Eqs. 12 and 13 we obtain the same result (up to a phase coming from our definition of $r_{-n}$ and $t_{-n}$ ) of ref. [6]: 


$$
\begin{aligned}
& r_{-n}=-i(\xi / 4)[\cos 2 \varphi+(\xi / 4) \sin 2 \varphi] t_{-n} \\
& t_{-n}=e^{-i k L}\left[(\xi / 4)^{2} e^{-2 i \varphi}+(1+i \xi / 4)^{2} e^{2 i \varphi}\right]^{-1} .
\end{aligned}
$$

For $\xi \gg 1$, we may use the series expansion for $J_{ \pm 1 / 3}\left(\xi^{-1} / 3\right)$ to obtain $\beta_{e}^{-n}=-\alpha k \xi^{1 / 3} \chi(\varphi)$ and $\beta_{o}^{-n}=$ $\alpha k \xi^{1 / 3} \chi(\varphi+\pi / 2)$ where $\alpha=(2 / 9)^{1 / 3} \Gamma(2 / 3) / \Gamma(4 / 3)$. In this case we get:

$$
r_{-n}=\frac{-i \alpha \xi^{1 / 3}(\chi(\varphi)+\chi(\varphi+\pi / 2))}{\left(1+i \alpha \xi^{1 / 3} \chi(\varphi)\right)\left(1-i \alpha \xi^{1 / 3} \chi(\varphi+\pi / 2)\right)} e^{-i k L}
$$

and

$$
t_{-n}=\frac{1-\alpha^{2} \xi^{2 / 3} \chi(\varphi) \chi(\varphi+\pi / 2)}{\left(1+i \alpha \xi^{1 / 3} \chi(\varphi)\right)\left(1-i \alpha \xi^{1 / 3} \chi(\varphi+\pi / 2)\right)} e^{-i k L} .
$$

Resonances occur when $\beta_{e}^{-n}$, or $\beta_{o}^{-n}$, are zero, that is when either $\chi(\varphi)$ or $\chi(\varphi+\pi / 2)$ are zero, which give us a simple condition for localizing them:

$$
\frac{\kappa_{n} L}{\pi} \int_{0}^{\pi / 2} \sqrt{\left(k / \kappa_{n}\right)^{2}+(\pi / 2) \cos (\theta)} d \theta=m \pi / 2+\pi / 12,
$$

with $m=0,1,2, \ldots$ For $\left(k / \kappa_{n}\right)^{2} \ll 1$ we may evaluate the integral approximately and get for the resonance positions:

$$
\kappa_{n} L \sim 2.092 \times(m \pi / 2+\pi / 12) .
$$

Therefore, for the sinusoidal field mode we will also obtain a series of resonances at values of $\kappa_{n} L$ that are separated by an interval approximately equal to $\pi$. In addition their positions are shifted in relation to the constant field case, their widths are wider and increase with $\kappa_{n} L$. We will show that these results give rise to new features in the physical quantities of interest.

\section{NUMERICAL RESULTS}

We have checked that the WKB-like eigenfunctions agrees extremely well with the numerical solutions at each point, even for low values of $\kappa_{n} L$ and $k / \kappa_{n}$. For higher values of $\kappa_{n} L$ (realistic values included) the WKBlike solution should be increasingly better.

For very small values of $\kappa_{n} L$ the resonances in $P_{\mathrm{em}}^{n}$, although wider than in the constant field case, do not correspond to appreciable values of $P_{\mathrm{em}}^{m}$ for $m \neq n$. For large values of $\kappa_{n} L$ the resonances in $P_{\mathrm{em}}^{n}$ are wide enough to produce appreciable values of $P_{\mathrm{em}}^{m}$ for $m \neq n$, so one should take them in account when predicting the total probabilities $P_{\mathrm{em}}$, or $T$. This is not the case for the constant field mode where the narrow resonances assure a similar behavior of $P_{\mathrm{em}}^{n}$ and $P_{\mathrm{em}}, T^{n}$ and $T$, except for accidental numerical coincidences.

In Fig. 1 we show our results for the probability of emission of a photon when there is zero photons inside the cavity (vacuum field), $P_{\mathrm{em}}^{o}$, for a fixed value of $k / \kappa_{n}=$ 0.01 , as a function of $\kappa_{n} L$ in the range $100 \pi<\kappa_{n} L<$ $104 \pi$. We see that the resonances are shifted and wider in the sinusoidal mode (solid line), in comparison with the constant mode (dashed line), and that their widths are still much smaller than the resonance separation.

We now consider that the cavity is in contact with a heat bath at a temperature associated with a thermal mean photon number $n_{b}$. Assuming, as usual, that we may treat gain and loss independently and that the interval of the atom separation obeys a Poissonian statistics, it can be shown that the stationary photon distribution is given by 3

$$
\begin{aligned}
& \frac{d p_{n}}{d t}=r\left(p_{n-1} P_{\mathrm{em}}^{n-1}-p_{n} P_{\mathrm{em}}^{n}\right)-\frac{\omega}{Q}\left(n_{b}+1\right) \\
& {\left[n p_{n}-(n+1) p_{n+1}\right]-\frac{\omega}{Q} n_{b}\left[(n+1) p_{n}-n p_{n+1}\right] .}
\end{aligned}
$$

Here $r$ is the atomic injection rate, $Q$ is the cavity quality factor, $\omega$ is the cavity resonance frequency and $n_{b}$ is the mean number of photons inside a cavity in thermal equilibrium with a reservoir at temperature $T_{b}$. From Eq. 31 we obtain the stationary population :

$$
p_{n}=p_{0} \prod \frac{n_{b}+N_{e x} P_{\mathrm{em}}^{j-1} / j}{\left(n_{b}+1\right)},
$$

where $N_{e x}=r Q / \omega$. This result is similar to that obtained in the conventional micromaser, where the value of $P_{\mathrm{em}}^{n}$ is given by $\sin ^{2}\left(\tau \kappa_{n}^{2} /(2 m)\right), \tau=(m / \hbar k) L$ being the time of flight of the atom through the cavity. For $k / \kappa_{0} \ll 1$ the photon distribution obtained from Eq. 32 is completely different from that of the conventional micromaser. In this case the predictions are strongly dependent on the field mode profile as long as $n_{b}$ is small enough and $N_{e x}$ large enough.

Fig. 2 shows the stationary photon distributions, for $N_{e x}=1000, n_{b}=1$ and $k / \kappa_{0}=0.01$, when the $\kappa_{2} L$ value corresponds to the position of the $100^{\text {th }}$ resonance in the case of the constant mode $\left(\kappa_{2} L=99 \pi\right)$ and of the sinusoidal mode $\left(\kappa_{2} L \approx 103.7 \pi\right)$. Fig 2 a (constant mode) shows the apparition of two thermal distributions with peaks at $n=3,12$. For such low values of $\kappa L$ this may happen in two special cases [6], when there is a resonance as in $\kappa L \sqrt[4]{3}=\left(99 \pi / 3^{1 / 4}\right)(3)^{1 / 4}=99 \pi$ $(n=3)$ or when there is a numerical coincidence as in $\kappa L \sqrt[4]{12}=\left(99 \pi / 3^{1 / 4}\right)(12)^{1 / 4} \approx 140.007 \pi(n=12)$. Otherwise we would obtain an equilibrium thermal distribution at the temperature corresponding to the average photon number $n_{b}$. This is not the case for the sinusoidal mode (see Fig. 2b) since the probability of photon emission, $P_{\mathrm{em}}^{j}$, may be important for several different values 
of $j$ besides the resonant probability for $n=0$, even at these low values of $\kappa L$. This is due to the large width of the resonances for the sinusoidal mode, in contrast with the sharp ones for the constant field case. As we mentioned before, these widths increase with $\kappa L$ for the sinusoidal mode, producing an extremely complex photon statistics.

Realistic values for the coupling of Rydberg atoms with microwave cavities are much larger than the values considered above. For example, consider the Rydberg transition between circular states with principal quantum numbers 50 and 51 in Rubidium at $51 \mathrm{GHz}$, used by the Ecole Normale Supérieure Group in recent experiments [12]. For a cylindrical cavity, with a length $L \approx 0.40 \mathrm{~cm}$ and a radius $R \approx 0.68 \mathrm{~cm}$, this transition is resonant with the $\mathrm{TE}_{121}$ mode. For a dipole moment $d \approx 10^{-26} \mathrm{Cm}$, we get $\kappa_{0} \approx 2.1 \times 10^{5} \mathrm{~cm}^{-1}$ and $\kappa_{0} L \approx 27000 \pi$.

In Fig. 3 we plot the probability of detecting one transmitted atom, $T^{n}=T_{e}^{n}+T_{f}^{n}$, and the probability that one photon be emitted, $P_{\mathrm{em}}^{n}=R_{f}^{n}+T_{f}^{n}$, if we have initially $n=0,1,2,3$ in the cavity, as a function of $\kappa_{0} L$, for $30000 \pi<\kappa_{0} L<30005 \pi$ and $k / \kappa_{0}=0.01$. This ratio corresponds to Rubidium atom velocities of approximately $0.2 \mathrm{~mm} / \mathrm{s}$. Atomic velocities lower than that have been already obtained by evaporative cooling [13. For these velocity values the injection rate should be very small, if we wish to have only one atom at a time in the cavity $\left(r \simeq 0.06 s^{-1}\right)$, and the value of $N_{e x}$ would be smaller than 1 even for $Q \approx 3 \times 10^{12}$ ! In this case the stationary photon distribution would be very close to a thermal one, inhibiting the new features shown in Fig. 2. In Fig. 3, after a careful inspection, we note that $T^{n}$ and $P_{\mathrm{em}}^{n}$ show a similar, but not identical, behavior. $P_{\mathrm{em}}^{n}$ presents better resolved resonances than $T^{n}$ and this could be useful for determining the more convenient experimental setup.

In Fig. 4 we plot the total probability of detecting the transmitted atom, $T$, for the sinusoidal mode (dotted line) and the constant mode (solid line), when a coherent state, either with $\bar{n}=0.25$ or $\bar{n}=2$, is initially present inside the cavity. The figure shows very sharp resonances in $\kappa_{0} L$ for the constant mode. They are a consequence of the fact that only one resonance is contributing for a given $n$, with a statistical weight $|c(n)|^{2}$. For the sinusoidal mode the resonances in $T^{n}$ are broader and several resonances may contribute for a fixed $\kappa_{0} L$. The case in which $\bar{n}=2$ is much more sensible to these features, showing, for the constant mode, many little resonance peaks and, for the sinusoidal mode, very bad resolved resonances.

\section{CONCLUSIONS}

The behavior of ultracold atoms interacting with a field inside a high quality microwave cavity depends in an essential way on the profile of the mode inside the cavity.
For warm atoms it is the value of the Rabi angle and the rate of incident atoms that defines the gain. For ultracold atoms we should also consider the exchange of momentum between the cavity and the atom. Most atoms are reflected when the atom-field system is in the state $|+n\rangle$, independently of the shape of the mode. When the atomfield system is in the state $|-n\rangle$ and the shape of the cavity is smooth enough, so that the effective de Broglie wavelength does not vary appreciably through the cavity, most atoms would be transmitted. When this is not the case the atoms would be reflected, unless $\kappa_{n} L$ is close to certain resonant values.

We have calculated, in the case of a sinusoidal mode, the probability of transmission of an ultracold atom and the probability of emission of a photon comparing them with the corresponding mesa function results. In the case of a mesa function sharp resonances appear in the transmission coefficient as a function of $\kappa_{n} L$. In the case of the sinusoidal mode resonances are present when the value of $\frac{\pi^{2}\left(\kappa_{n} / k\right)^{3}}{4 \kappa_{n} L} \gtrsim 1$, even when $\kappa_{n} L$ is very large. This may give us a chance to test these effects in a realistic experiment (when $\kappa_{0} L \sim 10^{5}$ ) using a cylindrical cavity. We also found, in the case of the sinusoidal mode, that the width of the resonances are larger, but still well resolved for realistic parameters, and increases with $\kappa_{0} L$, as long as $k / \kappa_{0}$ is very small. As a consequence, the transmission of atoms through a cavity containing a coherent state with few photons, as a function of $\kappa_{0} L$, has a completely different shape for the two modes we have studied. The broadening of the resonances produces also a more complex photon statistics for the stationary state, at least for theoretical parameters, to our knowledge, far from realistic ones.

These effects show the importance of considering the details of the cavity mode when analyzing the scattering of ultracold atoms by a resonant cavity.

We should remark that a serious difficulty in realizing these experiments is the long time of flight that ultracold atoms need to reach the cavity after their production.

\section{ACKNOWLEDGMENTS}

This work is supported in part by Centro Latino Americano de Física (CLAF), The Brazilian Conselho Nacional de Desenvolvimento Científico e Tecnológico (CNPq), Programa de Apoio a Núcleos de Excelência (PRONEX) and Fundação Universitária José Bonifácio (FUJB).

[1] See, for example, Laser Manipulation of Atoms and Ions, edited by E. Arimondo, W. D. Phillips and F. Strumia, Varenna on Lake Como Summer School ( North Holland, Amsterdan, 1992). 
[2] B. G. Englert, J. Schwinger, A. O. Barut and M. O. Scully, Europhysics Lett. 14, 25 (1991).

[3] M. O. Scully, G. M. Meyer, and H. Walther, Phys. Rev. Lett. 76, 4144 (1996).

[4] S. Haroche, M. Brune and J. M. Raimond Europhysics Lett. 14, 19 (1991).

[5] G. M. Meyer, M. O. Scully, H. Walther, Phys. Rev. A, 56, 4142 (1997).

[6] M. Löffler, G. M. Meyer, M. Schröder, M. O. Scully, H. Walther, Phys. Rev. A, 56, 4153 (1997).

[7] M. Schröder, K. Vogel, W. P. Schleich, M. O. Scully, H. Walther, Phys. Rev. A, 56, 4164 (1997).

[8] See, for example, P. Meystre, M. Sargent III in Elements of Quantum Optics, p. 446, Springer-Verlag, Berlin Heidelberg, 1990.

[9] See, for example, D. Jackson in Classical Eletromagnetism, p. 353, Addison-Wesley, New York (1975).

[10] J. H. Eberly, Am. Jour. Phys. 33, 771 (1965). Our phase shifts where defined with a different convention.

[11] See, for example, Morse and Feshbach in Methods of Theoretical Physics, ps. 1096-1106, Mc Graw-Hill, New York (1953).

[12] M. Brune, E. Hagley, J. Dreyer, X. Maitre, A. Maali, C. Wunderlich, J. M. Raimond and S. Haroche, Phys. Rev. Lett. 77, 4887 (1996).

[13] M. O. Mewes, M. R. Andrews, N. J. van Druten, D. M. Kurn, D. S. Durfee and W. Ketterle, Phys. Rev. Lett. 77, 416 (1996).

FIG. 1. Probability of emission of a photon when the cavity is initially empty as a function of $\kappa L$ when the mode is: the sinusoidal mode (solid line) and the constant mode (dotted line)

FIG. 2. Stationary photon distribution for $N_{e x}=1000$, $k / \kappa=0.01$ and $n_{b}=1$ when $\kappa_{2} L$ corresponds to the $100^{t h}$ resonance:(a)constant mode; (b) sinusoidal mode.

FIG. 3. (a)Probability that an atom being transmitted through the cavity and (b)Probability of emission of a photon when the cavity has initially $n$ photons, as a function of $\kappa L$ : $n=0$ (solid line), $n=1$ (dashed line), $n=2$ (dotted line).

$k / \kappa=0.01$. Sinusoidal mode

FIG. 4. Probability that an atom being transmitted when initially there is a coherent state in the cavity as a function of $\kappa L$ for the constant mode (full line) and the cosine mode (dashed line) (a) : $\bar{n}=0.25$ (solid line); (b): $\bar{n}=2$. $k / \kappa=0.01$. Sinusoidal mode 


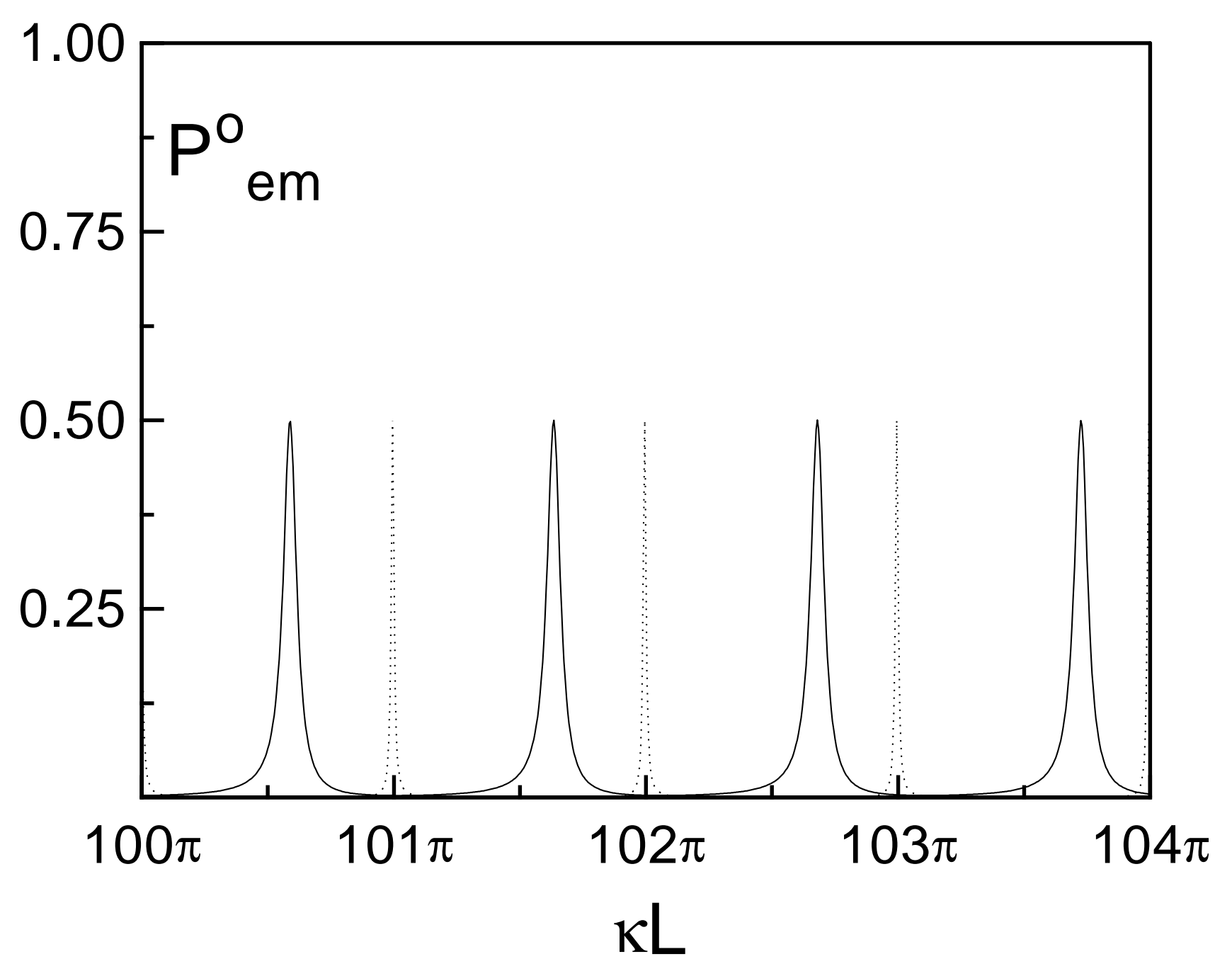

Figure 1: Retama 


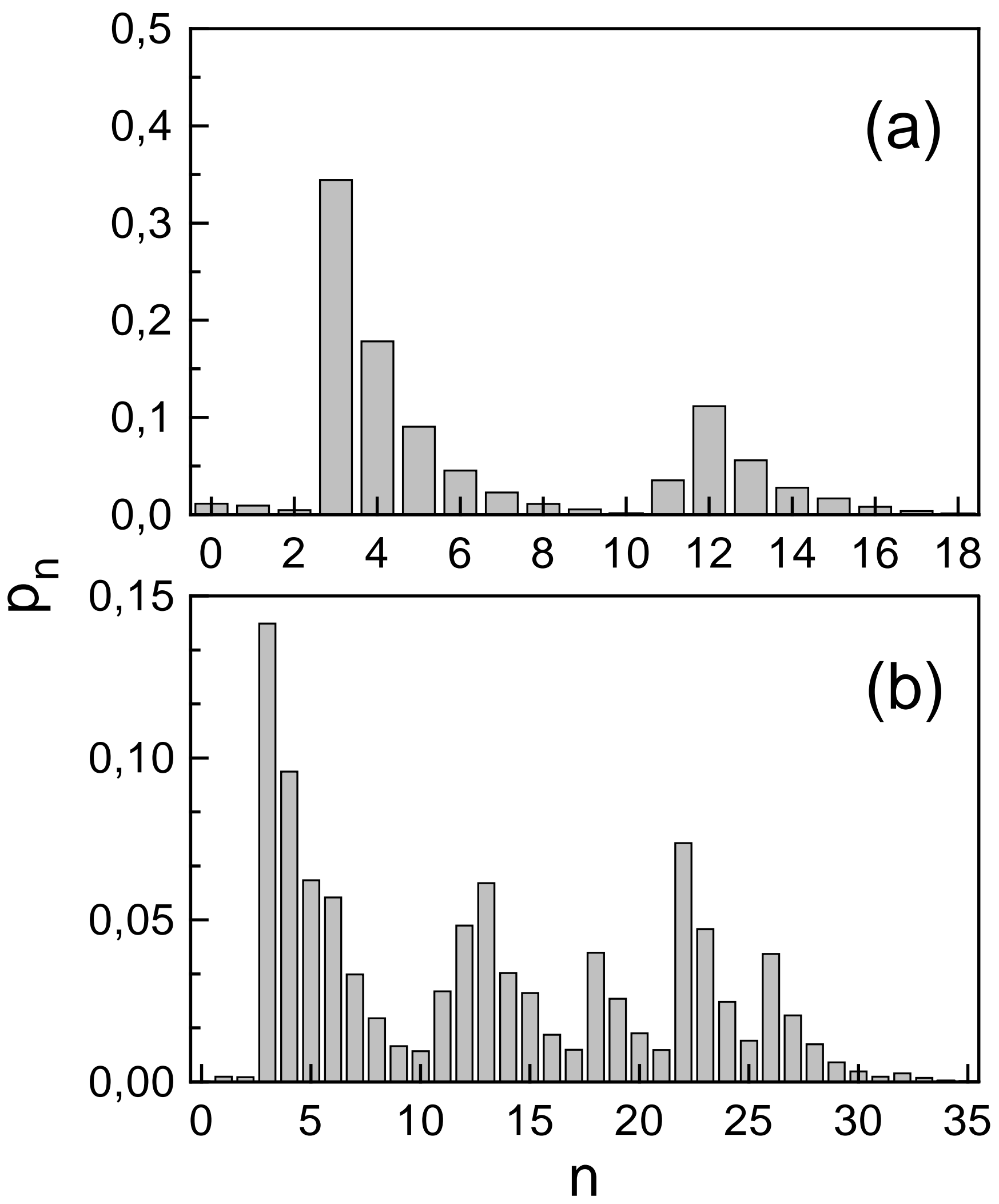

Figure 2: Retamal 

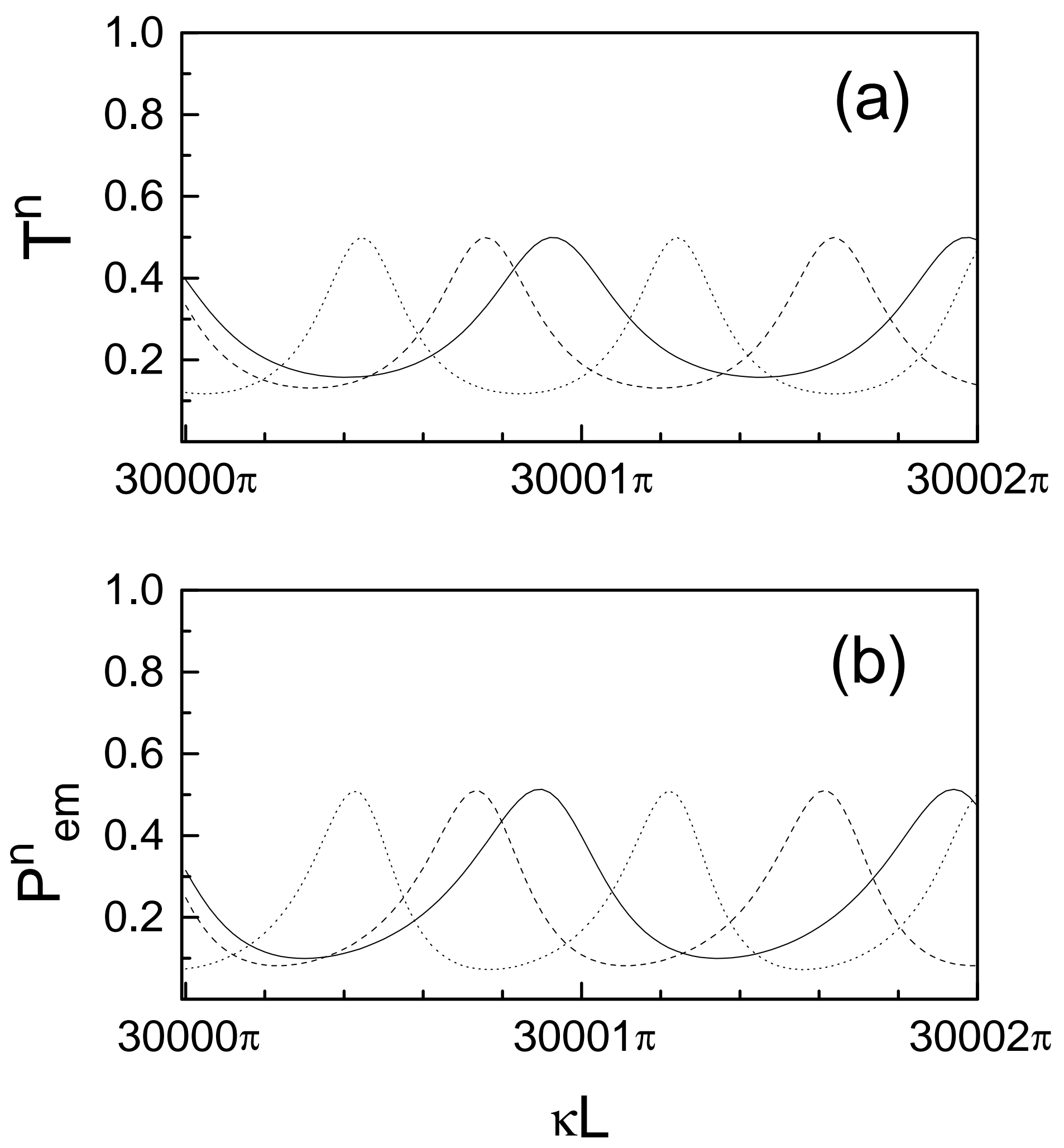

Figure 3: Retamal 


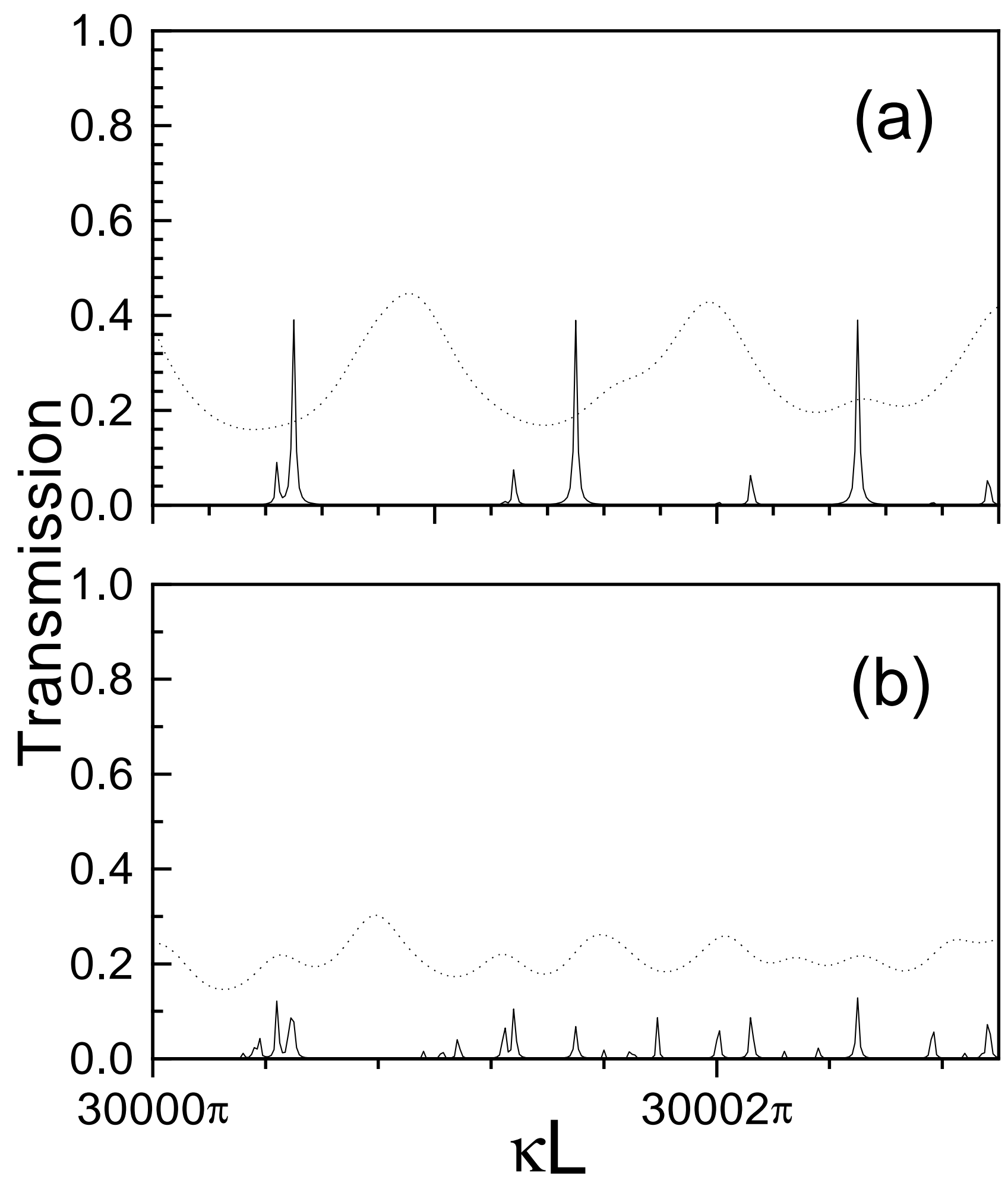

Figure 4: Retamal 